\title{
Caracterização da produção do conhecimento sobre sistematização da assistência de enfermagem no Brasil
}

\author{
CHARACTERIZATION OF KNOWLEDGE PRODUCTION ON THE PATIENT CARE SYSTEM IN BRAZIL \\ CARACTERIZACIÓN DE LAPRODUCCIÓN DEL CONOCIMIENTO SOBRE SISTEMATIZACIÓN \\ DE LAASISTENCIA DE ENFERMERÍAEN BRASIL
}

\author{
Rosely Moralez de Figueiredo', Silvia Helena Zem-Mascarenhas², \\ Anamaria Alves Napoleão ${ }^{3}$, André Bueno de Camargo ${ }^{4}$
}

\section{RESUMO}

Trata-se de um estudo bibliográfico que teve como objetivo caracterizar a produção científica nacional sobre o tema "Sistematização da Assistência de Enfermagem" e visualizar tendências da mesma. Foi realizado levantamento bibliográfico retrospectivo (2002-1990) das publicações, considerando os critérios: data, título do periódico, tipo de pesquisa e enfoque da publicação. A média da produção foi de 13,4 publicações/ano e os artigos distribuídos em 23 periódicos com predomínio de estudos realizados na área hospitalar (63,2\%). $\mathrm{O}$ tema cardiologia ocorreu em $17,6 \%$ das publicações e o foco da atenção na implementação, desenvolvimento e avaliação de modelos de SAE em 78,6\%. A taxonomia NANDA foi citada em $40,2 \%$ da produção e Wanda Horta em $40,5 \%$. Considera-se que existem ainda lacunas nessa área do conhecimento, mas a crescente divulgação da produção científica sobre o tema poderá auxiliar os profissionais na implementação da Sistematização da Assistência de Enfermagem.

\section{ABSTRACT}

This is a bibliographical study that has as its aim characterizing the Brazilian scientific production on the "Patient Care System" and visualizing its trends. A retrospective bibliogra-phical study (1990-2002) was carried out taking into consideration the items: date, title of the journal, type of research and approach of the publication. Production average was 13.4 publications/ year and articles were distributed among 23 journals, with a majority of them dealing with hospitals (63.2\%). Cardiology was the theme of $17.6 \%$ of the articles, and $78.6 \%$ of them were focused on the implementation, development and evaluation of SAE models. NANDA taxonomy was men-tioned in $40.2 \%$ of the production, and Wanda Horta in $40.5 \%$. There are still some blanks in this area of knowledge, but the increasing availability of the scientific production on this subject may help professionals in the implementation of the patient care system.

\section{RESUMEN}

Se trata de un estudio bibliográ-fico que tuvo como objetivo caracterizar la producción científica nacional sobre el tema "Sistematización de la Asistencia de Enfermería" y visualizar tendencias de la misma. Fue realizado un levantamiento bibliográfico retrospectivo (2002-1990) de las publicaciones, considerando los criterios: fecha, título del periódico, tipo de investigación y enfoque de la publicación. El promedio de la producción fue de 13,4 publicaciones/ año y los artículos distribuidos en 23 periódicos con predominio de estudios realizados en el área hospitalaria $(63,2 \%)$. El tema cardiología ocurrió en el 17,6\% de las publicaciones y el foco de la atención en la implementación, desarrollo y evaluación de modelos del SAE en el 78,6\%. La taxonomía de la NANDA fue citada en el 40,2\% de la producción y Wanda Horta en el $40,5 \%$. Se considera que existen aún lagunas en esa área del conocimiento, sin embargo la creciente divulgación de la producción científica sobre el tema puede auxiliar a los profesio-nales en la implementación de la Sistematización de la Asistencia de Enfermería.

\section{DESCRIPTORES}

Nursing process (trends).

Periodicals.

Nursing assessment.

Procesos de enfermería
1 Enfermeira. Professor Adjunto do Departamento de Enfermagem da Universidade Federal de São Carlos (UFSCar). rosely@power.ufscar.br

2 Enfermeira. Professor Adjunto do Departamento de Enfermagem da UFSCar. silviazem@ power.ufscar.br

3 Enfermeira. Professora Assistente do Departamento de Enfermagem da UFSCar. anamaria@ power.ufscar.br

4 Aluno do Curso de Enfermagem da UFSCar.

\section{DESCRITORES}

Processos de enfermagem (tendências).

Publicações periódicas.

Avaliação em enfermagem. (tendencias).

Publicaciones periódicas.

Evaluación en enfermería. 


\section{INTRODUÇÃO}

A preocupação em orientar as atividades de enfermagem com respaldo no método científico teve como marco o desenvolvimento e divulgação do Processo de Enfermagem, que foi inicialmente expresso na literatura norte americana, nas décadas de 1950 e $1960^{(1-3)}$.

No Brasil foi divulgado principalmente por Wanda Horta que publicou em 1979 um livro intitulado O Processo de Enfermagem.

O Processo de Enfermagem pode ser definido como um método através do qual a estrutura teórica da enfermagem é aplicada à prática ${ }^{(1)}$. Atualmente é apresentado em cinco fases: investigação, diagnóstico de enfermagem, planejamento, implementação e avaliação ${ }^{(4)}$.

Os assuntos relativos a esta temática continuam a constituir, atualmente, objeto de preocupação de enfermeiros em diferentes âmbitos de atuação, quais sejam, ensino, pesquisa e assistência.

A legislação brasileira, através da Lei do Exercício Profissional, Lei ${ }^{\circ} 7498 / 86$, em seu artigo $8^{\circ}$, dispõe que $a o$ enfermeiro incumbe (...) a participação na elaboração, execução e avaliação dos planos assistenciais de saúde $(\ldots)^{(5)}$. Sistematizar, individualizar, administrar e assumir o papel de prestador do cuidado de enfermagem junto à equipe, são metas e desejos que as enfermeiras têm demonstrado em encontros da categoria ${ }^{(2)}$. Carraro et al consideram que a cientificação profissional é almejada pelos profissionais de enfermagem e só será alcançada com a utilização de instrumentos científicos que subsidiem a prática profissional ${ }^{(6)}$.

Recentemente o termo sistematização da assistência de enfermagem (SAE) foi incluído na Decisão COREn-SP/DIR/ 008/1999. Esta decisão normatiza a implementação da sistematização da assistência de enfermagem do Conselho Regional de Enfermagem - SP e prevê como atividade privativa do Enfermeiro a implantação, planejamento, organização, execução e avaliação do Processo de Enfermagem ${ }^{(7)}$.

Na década de 1990 com a publicação da Lei de Diretrizes e Bases da Educação (Lei n’ 9394/96) ocorreu a substituição do antigo currículo mínimo pelas diretrizes curriculares, trazendo maior flexibilização à organização e à operacionalização do currículo pleno de graduação. Desta forma o processo de enfermagem pôde ser inserido de forma mais efetiva nos currículos $^{(8)}$. Isso vem contribuindo para a formação de uma massa crítica no assunto.

Mesmo com o empenho do Conselho e de toda a classe profissional trata-se de um conhecimento que, apesar de ter sido introduzido no Brasil na década de 1970, ainda apresenta uma enorme lacuna entre a produção do conhecimento e sua aplicabilidade na prática diária do enfermeiro.

Estudos que avaliam a produção literária em diversas áreas da enfermagem são freqüentemente utilizados com o objetivo de apontar lacunas no conhecimento e direcionar trabalhos futuros ${ }^{(9-12)}$. Assim sendo, objetivando caracterizar a bibliografia nacional sobre o tema "sistematização da assistência de enfermagem" e visualizar as tendências da mesma, é que se propõe este trabalho.

\section{MATERIAL E MÉTODO}

Trata-se de um estudo bibliográfico sobre o tema sistematização da assistência de enfermagem no Brasil.

Foi realizado levantamento bibliográfico retrospectivo, do período 2002-1990, por meio do banco de dados LILACS (Literatura Latino-Americana e do Caribe em Ciências da Saúde), utilizando-se as palavras-chaves sistematização da assistência de enfermagem, processo de enfermagem, diagnóstico de enfermagem e NANDA (North American Nursing Diagnosis Association $\left.{ }^{(13)}\right)$ em artigos publicados em periódicos brasileiros.

Os dados foram armazenados em banco de dados informatizado e para análise das publicações consideramos os critérios: data, título do periódico, tipo de pesquisa (estudo de campo ou estudo teórico) e enfoque da publicação (hospitalar, extra-hospitalar ou outros).

A seleção do artigo se deu por meio da leitura do título e resumo de todos aqueles levantados na base de dados. Foram então excluídos os artigos que na leitura do resumo não apresentaram relação com o tema em questão.

\section{RESULTADOS E DISCUSSÃO}

Foram levantados 189 artigos e após a leitura do resumo 15 foram excluídos por não apresentaram relação com o tema.

A média obtida na distribuição dos 174 artigos selecionados pelo período é de 13,4 publicações/ano. $\mathrm{O}$ ano de 2002 destaca-se da média, apresentando 25 publicações (Figura 1). Esta elevação pode ser reflexo de mudanças como interesse maior em relação à temática por parte das instituições e o crescente envolvimento de enfermeiros, professores e pesquisadores com o tema, o que subsidia a reflexão, o ensino e a produção de conhecimento na área. Talvez uma outra hipótese a ser considerada seja a inclusão de novas revistas de enfermagem em bases de dados informatizadas, o que permitiu a localização do artigo neste trabalho. 


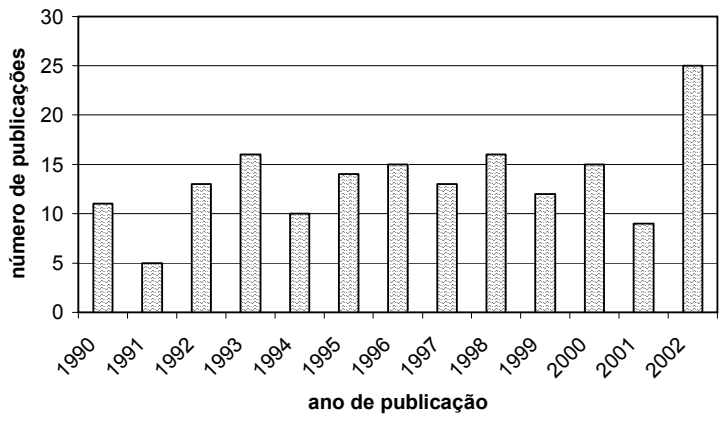

Figura 1 - Distribuição dos artigos de acordo com o ano de publicação - Brasil - 1990-2002
As publicações identificadas estão distribuídas em 23 periódicos sendo que $23,6 \%$ dos artigos são da Revista Latino-Americana de Enfermagem, $14,9 \%$ da Revista da Escola de Enfermagem da USP e 14,4\% da Revista Brasileira de Enfermagem (Quadro 1). Observa-se uma concentração de artigos nas revistas ligadas a escolas de enfermagem paulistas, talvez por serem estes grandes centros de pósgraduação e, portanto geradores de conhecimento.

Quadro 1 - Distribuição das publicações segundo o periódico - Brasil - 1990-2002

\begin{tabular}{|c|c|c|}
\hline Periódico & $\begin{array}{l}\text { Número de } \\
\text { Publicações }\end{array}$ & $\%$ \\
\hline Acta Paul. Enfermagem & 13 & 7,5 \\
\hline Braz. J. Epilepsy Clin. Neurophysiol & 1 & 0,6 \\
\hline CCS & 3 & 1,7 \\
\hline Cogitare Enferm & 3 & 1,7 \\
\hline Esc Anna Nery Rev Enfermagem & 2 & 1,1 \\
\hline Mundo Saúde & 1 & 0,6 \\
\hline Neurobiologia & 1 & 0,6 \\
\hline Nursing (São Paulo) & 3 & 1,7 \\
\hline Rev. Baiana de Enfermagem & 5 & 2,9 \\
\hline Rev. Bras. Cancerol & 1 & 0,6 \\
\hline Rev. Bras. Cienc. Saude & 2 & 1,1 \\
\hline Rev. Bras. Enfermagem & 25 & 14,4 \\
\hline Rev. Enfermagem UERJ & 13 & 7,5 \\
\hline Rev. Esc. Enfermagem USP & 26 & 14,9 \\
\hline Rev. Gaúch. Enfermagem & 11 & 6,3 \\
\hline Rev. HCPA \& Fac. Med. Univ. Fed. Rio Gd. do Sul & 1 & 0,6 \\
\hline Rev. Latino-Am. Enfermagem & 41 & 23,6 \\
\hline Rev. Paul. Enfermagem & 8 & 4,6 \\
\hline Rev. Saude Dist. Fed & 2 & 1,1 \\
\hline Rev. Soc. Cardiol. Estado de São Paulo & 6 & 3,4 \\
\hline Semina & 1 & 0,6 \\
\hline Texto Contexto Enfermagem & 5 & 2,9 \\
\hline
\end{tabular}

Outro aspecto levantado foi o enfoque dado pelas publicações onde $63,2 \%$ refere-se a estudos realizados em área hospitalar e $15,5 \%$ extra-hospitalar. Em $21,3 \%$ das publicações esta classificação não pôde ser adotada por se tratar de estudos teóricos, bibliográficos ou o enfoque não ter sido explicitado no resumo (Figura 2).

O predomínio do enfoque hospitalar pode estar relacionado à própria Lei do Exercício Profissional que preconiza que a sistematização da assistência de enfermagem seja priorizada para os pacientes com risco de vida e que exijam tomadas de decisões imediatas ${ }^{(7)}$, o que acontece predominantemente na área hospitalar.

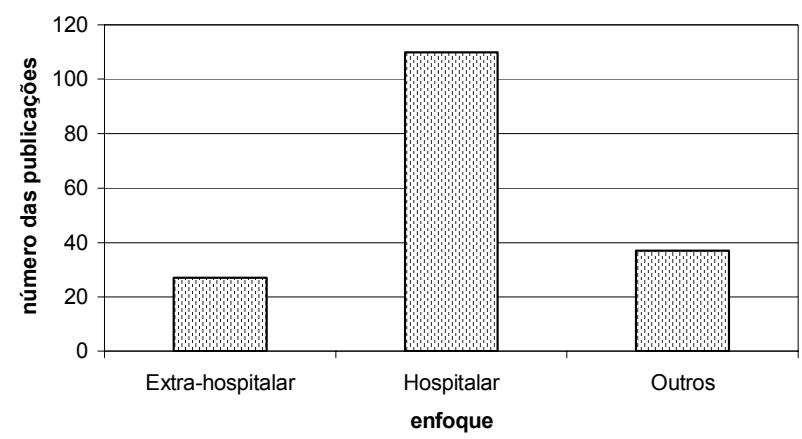

Figura 2 - Distribuição das publicações de acordo com o enfoque - Brasil - 1990-2002 
Em relação à área (especialidade) de desenvolvimento dos estudos, em $62,2 \%$ dos trabalhos foi possível identificála e nestes $17,6 \%$ referia-se à área de cardiologia, $14,8 \%$ à de perioperatório e 12,0\% à da mulher (Quadro 2).

Quadro 2 - Distribuição das publicações segundo área mencionada no resumo - Brasil - 1990-2002

\begin{tabular}{|l|c|c|}
\hline \multicolumn{1}{|c|}{ Área (Especialidade) } & $\begin{array}{c}\text { Número de } \\
\text { Publicações }\end{array}$ & $\mathbf{\%}$ \\
\hline Cardiologia & 19 & 17,6 \\
Perioperatório & 16 & 14,8 \\
Unidade de Terapia Intensiva & 11 & 10,2 \\
Mulher & 13 & 12,0 \\
Oncologia & 12 & 11,1 \\
Queimados & 7 & 6,5 \\
Neurologia & 7 & 6,5 \\
Idoso & 5 & 4,6 \\
Saúde Mental & 5 & 4,6 \\
Pediatria & 4 & 3,7 \\
Outros & 9 & 8,3 \\
\hline
\end{tabular}

Em 131 (75,3\%) artigos selecionados o foco de atenção da publicação pôde ser identificado. A implementação, o desenvolvimento e a avaliação de modelos de SAE surgiram como foco em 78,6\% dos trabalhos. Isso endossa os achados obtidos na classificação entre estudo teórico ou de campo, onde $81,0 \%$ dos trabalhos foram classificados como sendo de campo (Figura 3 ).

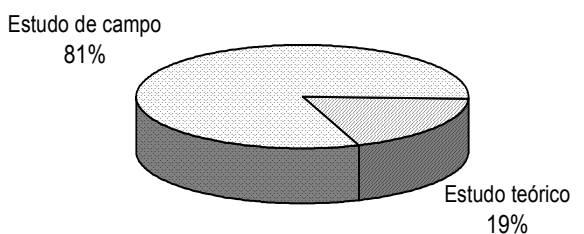

Figura 3 - Distribuição das publicações segundo o tipo de pesquisa - Brasil - 1990-2002

Na literatura brasileira da década de 1980 já se encontravam vários relatos sobre experiências de implementação do processo de enfermagem em instituições de saúde brasileiras, o que reforça a existência de um interesse antigo, por parte dos enfermeiros, em operacionalizar o processo ${ }^{(14)}$. Entretanto, quando levantada a produção bibliográfica do período deste trabalho, observa-se que a operacionalização da SAE ainda continua sendo o principal foco da produção. Pode-se depreender disso que o processo de sistematização da assistência de enfermagem no Brasil continua em fase de construção, procurando-se caminhos e estratégias que sejam aplicáveis nas diferentes áreas de atuação profissional.

Alegações como falta de profissionais e área de conhecimento nova aparecem como justificativas para as dificuldades na implementação da SAE no Brasil ${ }^{(15)}$. Entretanto não foram identificados, nas publicações selecionadas, tra- balhos que discutissem de maneira mais ampla outros fatores que poderiam estar dificultando a implementação do processo nas diferentes instituições brasileiras como: relação de poder dentro das instituições, organização do trabalho e falta de autonomia do enfermeiro.

No que tange à distribuição das publicações segundo o tipo de pesquisa, em $70(40,2 \%)$ delas houve menção à utilização da taxonomia NANDA. A taxonomia NANDA foi o primeiro sistema de classificação para diagnósticos de enfermagem disponível em português, tendo sido traduzida e modificada por Nóbrega e Garcia em 1994 ${ }^{(16)}$. Isto pode explicar a maior divulgação deste sistema no Brasil.

Quanto a modelos teóricos 37 (21,2\%) publicações o identificam, dentre essas Wanda Horta aparece em 15 (40,5\%) publicações, seguida por Roy (16,2\%), Oren e Levine (10,2\%) cada, Roger e King (5,2\%) cada, Orlando, Dorothy Johnson, Baley \& Claus, Irving e Kurt Lewin com apenas (2,5\%) cada um. Em outro estudo ${ }^{(8)}$ Wanda Horta foi referida como suporte teórico por $52 \%$ dos docentes entrevistados que ministravam os conteúdos sobre processo de enfermagem, fato considerado coerente pelas autoras, tendo em vista ser este modelo teórico o mais conhecido no Brasil.

A escolha de um referencial teórico e subsidiá-lo na prática assistencial, no ensino ou na pesquisa implica identificar o paradigma adotado, distinguindo-o daqueles adotados por outras pessoas ${ }^{(17)}$. Esta escolha também deveria ser feita em consenso com os objetivos da instituição e da equipe, de forma a encontrar o melhor suporte teórico para aquilo a que se propõe com a SAE.

\section{CONSIDERAÇÕES FINAIS}

O estudo permitiu um panorama da produção científica brasileira sobre o tema Sistematização da Assistência de Enfermagem no período de 1990-2002.

A média da produção é de 13,4 publicações/ano, com destaque ao ano de 2002 que apresentou 25 publicações. Os artigos estão distribuídos em 23 periódicos. Duas revistas ligadas a grandes universidades paulistas concentram 39\% da produção do período.

Observa-se um predomínio da produção na área hospitalar com $63,2 \%$, na área de cardiologia com $17,6 \%$ e com o foco da atenção na implementação, no desenvolvimento e na avaliação de modelos de SAE em 78,6\% dos artigos.

A taxonomia NANDA foi citada em $40,2 \%$ da produção e dentre as obras que fizeram menção a modelos teóricos Wanda Horta apareceu em 40,5\% deles.

Considera-se que existem ainda muitas lacunas na produção de conhecimento sobre o tema, em especial na análi- 
se das características administrativas das instituições de saúde, na posição e autonomia da enfermagem, na concentração do poder dentro das instituições e sua interferência na implementação da SAE.

\section{REFERÊNCIAS}

(1) Iyer PW, Taptich BJ, Bernocchi-Losey D. Processo e diagnóstico em enfermagem. Porto Alegre: Artes Médicas; 1993.

(2) Campedelli MC. Processo de enfermagem na prática. São Paulo: Ática; 1989.

(3) Horta WA. Processo de enfermagem. São Paulo: EPU; 1979.

(4) Alfaro-Lefreve R. A aplicação do processo de enfermagem: um guia passo a passo. $4^{\mathrm{a}}$ ed. Porto Alegre: Artes Médicas; 2000.

(5) Brasil. Lei n. 7498, de 25 de Junho de 1986. Dispõe sobre a regulamentação do exercício da enfermagem e dá outras providências. Diário Oficial da República Federativa do Brasil, Brasília, 26 jun. 1986. Seção 1; p. 1.

(6) Carraro TE, Kletemberg DF, Gonçalves LM. O ensino da metodologia da assistência de enfermagem no Paraná. Rev Bras Enferm. 2003;56(5):499-501.

(7) Conselho Regional de Enfermagem-São Paulo. Decisão COREnSP-DIR/008/1999 "Normatiza a implementação da sistematização da assistência de enfermagem - SAE - nas Instituições de Saúde, no Âmbito do Estado de São Paulo". [online] Disponível em: http://www.corensp.org.br/resolucoes/decisoes.html> [Acesso em 10 dez. 2003].

(8) Dell‘Acqua MCQ, Miyadahara AMK. Ensino do processo de enfermagem nas escolas de graduação em enfermagem do estado de São Paulo. Rev Lat Am Enferm. 2002;10(2):185-91.

(9) Carvalho IMP, Melo RL, Andraus LMS. Produção científica de enfermagem em nefrologia, no Brasil, no período de 1989 até 1999. Rev Eletron Enferm. [periódico online] 2001;3(2). Disponível em: http://www.fen.ufg.br/revista $>$ [Acesso em 20 abr. 2004].
Acredita-se que trabalhos que analisem esta interface entre os diferentes modelos administrativos das instituições e a implementação da SAE devam ser incentivados.

(10) Reis RK, Gir E. Caracterização da produção científica sobre doenças sexualmente transmissíveis e HIV/AIDS publicados em periódicos de enfermagem do Brasil. Rev Esc Enferm USP. 2002;36(4):76-85.

(11) Marziali MHP, Rodrigues CM. A produção científica sobre os acidentes de trabalho com material pérfurocortante entre trabalhadores de enfermagem. Rev Lat Am Enferm. 2002;10(4):571-7.

(12) Zem-Mascarenhas SH, Carvalho EC de. Toque Terapêutico: análise da produção do conhecimento utilizando uma base de dados informatizadas. REME - Rev Min Enferm. 1988; 2(2):98-103.

(13) North American Nursing Diagnosis Association (NANDA). Diagnósticos de enfermagem 2001/2002. São Paulo: Artmed; 2001.

(14) Rossi LA, Casagrande LDR. O processo de enfermagem em uma unidade de queimados: um estudo etnográfico. Rev Lat Am Enferm. 2001;9(5):39-46.

(15) Menossi MJ, Gilbert MJ, Nóbrega CRB, Rossi F, Carvalho $\mathrm{H}$, Ceribelli C, et al. A implantação da sistematização da assistência de enfermagem em uma unidade pediátrica: estudo piloto. Rev Soc Bras Enferm Pediatr. 2002;1(2):7-20.

(16) Vale IN, Lopes MHBM. A utilização de classificações na prática e no ensino de enfermagem: a experiência da UNICAMP. Rev Bras Enferm. 2002;55(6):670-3.

(17) Arreguy-Sena C, Carvalho EC, Rossi LA, Caron-Ruffino M. Estratégias de implantação do processo de enfermagem para uma pessoa infectada pelo HIV. Rev Lat Am Enferm. 2001;9(1):27-38. 


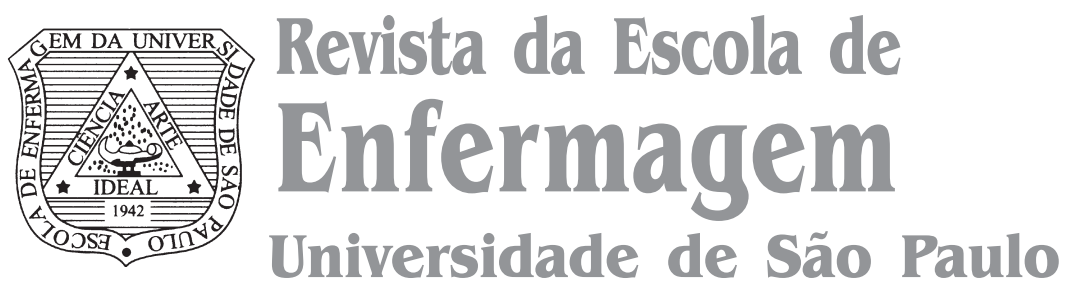

ERRATA

Como publicado no volume 40, número 2 de 2006, página 299, o nome do quarto autor está incorreto. Onde lê-se "Sueli Aparecida Frari Galera", leia-se: "André Bueno de Camargo".

A citação correta em referência é:

Figueiredo RM, Zem-Mascarenhas SH, Napoleão AM, Camargo AB. Caracterização da produção do conhecimento sobre sistematização da assistência de enfermagem no Brasil. Rev Esc Enferm USP. 2005;40(2):299-303. 
O arquivo disponível sofreu correções conforme ERRATA publicada no Volume 40 Número 3 da revista. 